\title{
2020 MMOR best paper award
}

\section{Oliver Stein ${ }^{1}$}

Accepted: 11 March 2021 / Published online: 16 April 2021

(C) The Author(s) 2021

Each year the MMOR Best Paper Award is bestowed on an excellent article published online in MMOR during the previous year. The list of past and current awardees is published on the MMOR web page. Candidates are nominated by the members of the MMOR editorial board, and the editorial board decides about the awardee(s).

In 2020 we published 32 papers online in MMOR. It is my great pleasure to announce that the 2020 MMOR Best Paper Award has been bestowed on the paper

\section{Interplay of non-convex quadratically constrained problems with adjustable robust optimization}

by Immanuel Bomze and Markus Gabl

MMOR 93 (2021), 115-151, published October 6, 2020.

Abstract: In this paper we explore convex reformulation strategies for non-convex quadratically constrained optimization problems (QCQPs). First we investigate such reformulations using Pataki's rank theorem iteratively. We show that the result can be used in conjunction with conic optimization duality in order to obtain a geometric condition for the S-procedure to be exact. Based upon known results on the S-procedure, this approach allows for some insight into the geometry of the joint numerical range of the quadratic forms. Then we investigate a reformulation strategy introduced in recent literature for bilinear optimization problems which is based on adjustable robust optimization theory. We show that, via a similar strategy, one can leverage exact reformulation results of QCQPs in order to derive lower bounds for more complicated quadratic optimization problems. Finally, we investigate the use of reformulation strategies in order to derive characterizations of set-copositive matrix cones. Empirical evidence based upon first numerical experiments shows encouraging results.

Oliver Stein

stein@kit.edu

1 Institute of Operations Research, Karlsruhe Institute of Technology (KIT), Karlsruhe, Germany 


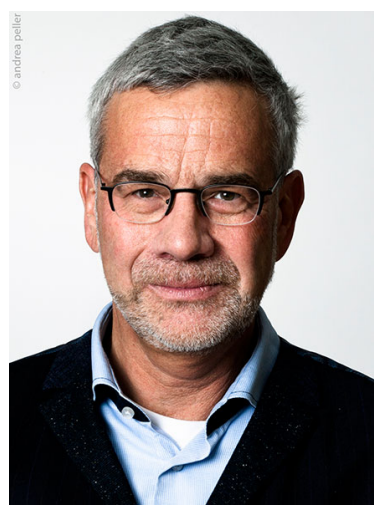

Since 2004, Immanuel M. Bomze holds a chair of Applied Mathematics and Statistics at the University of Vienna. Bomze's research interests are in the areas of Operations Research and Data Science, in particular nonlinear optimization and mathematical modeling, where he has edited one and published four books, as well as about 120 peer-reviewed articles in scientific journals and monographs. The list of his co-authors comprises nearly 100 scientists from more than a dozen countries in four continents. In 2014 he was elected Fellow of EurOpt, 2011-2017 he served as Co-Editor-in-Chief of EJOR, 2018-2020 as EURO president, and from 2021 he is the Editor-in-Chief of EJCO.

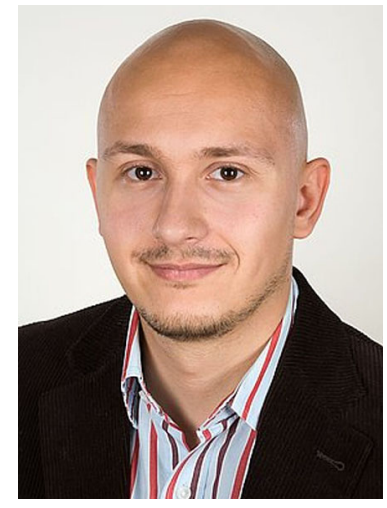

Markus Gabl is Ph.D. student at the Vienna Graduate School on Computational Optimization since 2016. His research interests comprise applications of copositve programming approaches, such as quadratic and robust programming, as well as applications of semidefinite programming in finance.

Congratulations in the name of the MMOR editorial board! 


\section{Funding Open Access funding enabled and organized by Projekt DEAL.}

Open Access This article is licensed under a Creative Commons Attribution 4.0 International License, which permits use, sharing, adaptation, distribution and reproduction in any medium or format, as long as you give appropriate credit to the original author(s) and the source, provide a link to the Creative Commons licence, and indicate if changes were made. The images or other third party material in this article are included in the article's Creative Commons licence, unless indicated otherwise in a credit line to the material. If material is not included in the article's Creative Commons licence and your intended use is not permitted by statutory regulation or exceeds the permitted use, you will need to obtain permission directly from the copyright holder. To view a copy of this licence, visit http://creativecommons.org/licenses/by/4.0/.

Publisher's Note Springer Nature remains neutral with regard to jurisdictional claims in published maps and institutional affiliations. 